\title{
Mappings of type Orlicz and generalized Cesáro sequence space
}

\author{
Nashat F Mohamed ${ }^{1 *}$ and Awad A Bakery ${ }^{1,2}$
}

${ }^{\text {*Correspondence: }}$

n_faried@hotmail.com

'Department of Mathematics, Faculty of Science, Ain Shams

University, Cairo, Egypt

Full list of author information is

available at the end of the article

\begin{abstract}
We study the ideal of all bounded linear operators between any arbitrary Banach spaces whose sequence of approximation numbers belong to the generalized Cesáro sequence space and Orlicz sequence space $\ell_{M}$, when $\mathbf{M}(t)=t^{p}, 0<p<\infty$; our results coincide with that known for the classical sequence space $\ell_{p}$.
\end{abstract}

Keywords: approximation numbers; operator ideal; generalized Cesáro sequence space; Orlicz sequence space

\section{Introduction}

By $L(X, Y)$, we denote the space of all bounded linear operators from a normed space $\mathrm{X}$ into a normed space $Y$. The set of natural numbers will denote by $\mathbb{N}=\{0,1,2, \ldots\}$ and the real numbers by $\mathbb{R}$. By $\omega$, we denote the space of all real sequences. A map which assigns to every operator $T \in L(X, Y)$ a unique sequence $\left(s_{n}(T)\right)_{n=0}^{\infty}$ is called an $s$-function and the number $s_{n}(T)$ is called the $n$th $s$-numbers of $T$ if the following conditions are satisfied:

(a) $\|T\|=s_{0}(T) \geq s_{1}(T) \geq \cdots \geq 0$, for all $T \in L(X, Y)$.

(b) $s_{n+m}\left(T_{1}+T_{2}\right) \leq s_{n}\left(T_{1}\right)+\left\|T_{2}\right\|$, for all $T_{1}, T_{2} \in L(X, Y)$.

(c) $s_{n}(R S T) \leq\|R\| s_{n}(S)\|T\|$, for all $T \in L\left(X_{0}, X\right), S \in L(X, Y)$ and $R \in L\left(Y, Y_{0}\right)$.

(d) $s_{n}(\lambda T)=|\lambda| s_{n}(T)$, for all $T \in L(X, Y), \lambda \in \mathbb{R}$.

(e) $\operatorname{rank}(T) \leq n$ If $s_{n}(T)=0$, for all $T \in L(X, Y)$.

(f) $s_{r}\left(I_{n}\right)=\left\{\begin{array}{ll}1 & \text { for } r<n, \\ 0 & \text { for } r \geq n,\end{array}\right.$, where $I_{n}$ is the identity operator on the Euclidean space $\ell_{2}^{n}$.

Example of $s$-numbers, we mention approximation number $\alpha_{r}(T)$, Gelfand numbers $c_{r}(T)$, Kolmogorov numbers $d_{r}(T)$ and Tichomirov numbers $d_{n}^{*}(T)$ defined by:

(I) $\alpha_{r}(T)=\inf \{\|T-A\|: A \in L(X, Y)$ and $\operatorname{rank}(A) \leq r\}$.

(II) $c_{r}(T)=a_{r}\left(J_{Y} T\right)$, where $J_{Y}$ is a metric injection (a metric injection is a one to one operator with closed range and with norm equal one) from the space $Y$ into a higher space $\ell^{\infty}(\Lambda)$ for suitable index set $\Lambda$.

(III) $d_{n}(T)=\inf _{\operatorname{dim} Y \leq n} \sup _{\|x\| \leq 1} \inf _{y \in Y}\|T x-y\|$.

(IV) $d_{r}^{*}(T)=d_{r}\left(J_{Y} T\right)$.

All of these numbers satisfy the following condition:

(g) $s_{n+m}\left(T_{1}+T_{2}\right) \leq s_{n}\left(T_{1}\right)+s_{m}\left(T_{2}\right)$ for all $T_{1}, T_{2} \in L(X, Y)$.

An operator ideal $U$ is a subclass of $L=\{L(X, Y) ; X, Y$ are Banach spaces $\}$ such that its components $\{U(X, Y) ; X, Y$ are Banach spaces $\}$ satisfy the following conditions:

(i) $I_{K} \in U$, where $K$ denotes the 1-dimensional Banach space, where $U \subset L$.

(ii) If $T_{1}, T_{2} \in U(X, Y)$, then $\lambda_{1} T_{1}+\lambda_{2} T_{2} \in U(X, Y)$ for any scalars $\lambda_{1}, \lambda_{2}$.

(iii) If $V \in L\left(X_{0}, X\right), T \in U(X, Y), R \in L\left(Y, Y_{0}\right)$ then $R T V \in U\left(X_{0}, Y_{0}\right)$. See [1-3]. 
An Orlicz function is a function $M:[0, \infty[\rightarrow[0, \infty[$ which is continuous, nondecreasing and convex with $M(0)=0$ and $M(x)>0$ for $x>0$, and $M(x) \rightarrow \infty$ as $x \rightarrow \infty$. See $[4,5]$.

If convexity of Orlicz function $M$ is replaced by $M(x+y) \leq M(x)+M(y)$. Then this function is called modulus function, introduced by Nakano [6]; also, see [7, 8] and [9]. An Orlicz function $M$ is said to satisfy $\Delta_{2}$-condition for all values of $\mathrm{u}$, if there exists a constant $k>0$, such that $M(2 u) \leq k M(u)(u \geq 0)$. The $\Delta_{2}$-condition is equivalent to $M(l u) \leq k l M(u)$ for all values of $\mathrm{u}$ and for $l>1$. Lindentrauss and Tzafriri [10] used the idea of Orlicz function to construct Orlicz sequence space

$$
\ell_{M}=\left\{x \in \omega: \sum_{n=0}^{\infty} M\left(\frac{\left|x_{n}\right|}{\rho}\right)<\infty, \text { for some } \rho>0\right\},
$$

which is a Banach space with respect to the norm

$$
\|x\|=\inf \left\{\rho>0: \sum_{n=0}^{\infty} M\left(\frac{\left|x_{n}\right|}{\rho}\right) \leq 1\right\} .
$$

For $\mathrm{M}(t)=t^{p}, 1 \leq p<\infty$ the space $\ell_{M}$ coincides with the classical sequence space $\ell_{p}$. Recently, different classes of sequences have been introduced by using an Orlicz function. See [11] and [12].

Remark 1.1 Let $M$ be an Orlicz function then $M(\lambda x) \leq \lambda M(x)$ for all $\lambda$ with $0<\lambda<1$.

For a sequence $p=\left(p_{n}\right)$ of positive real numbers with $p_{n} \geq 1$, for all $n \in \mathbb{N}$ the generalized Cesáro sequence space is defined by

$$
\operatorname{Ces}\left(p_{n}\right)=\left\{x=\left(x_{k}\right) \in \omega: \rho(\lambda x)<\infty \text { for some } \lambda>0\right\},
$$

where

$$
\rho(x)=\sum_{n=0}^{\infty}\left(\frac{1}{n+1} \sum_{k=0}^{n}\left|x_{k}\right|\right)^{p_{n}} .
$$

The space $\operatorname{Ces}\left(p_{n}\right)$ is a Banach space with the norm

$$
\|x\|=\inf \left\{\lambda>0: \rho\left(\frac{x}{\lambda}\right) \leq 1\right\} .
$$

If $p=\left(p_{n}\right)$ is bounded, we can simply write

$$
\operatorname{Ces}\left(p_{n}\right)=\left\{x \in \omega: \sum_{n=0}^{\infty}\left(\frac{1}{n+1} \sum_{k=0}^{n}\left|x_{k}\right|\right)^{p_{n}}<\infty\right\} .
$$

Also, some geometric properties of $\operatorname{Ces}\left(p_{n}\right)$ are studied by Sanhan and Suantai [13].

Throughout this paper, the sequence $\left(p_{n}\right)$ is a bounded sequence of positive real numbers, we denote $e_{i}=(0,0, \ldots, 1,0,0, \ldots)$ where 1 appears at $i$ th place for all $i \in \mathbb{N}$. Different classes of paranormed sequence spaces have been introduced and their different properties have been investigated. See [14-18] and [19]. 
For any bounded sequence of positive numbers $\left(p_{k}\right)$, we have the following well-known inequality $\left|a_{k}+b_{k}\right|^{p_{k}} \leq 2^{h-1}\left(\left|a_{k}\right|^{p_{k}}+\left|b_{k}\right|^{p_{k}}\right), h=\sup _{n} p_{n}$, and $p_{k} \geq 1$ for all $k \in \mathbb{N}$. See [20].

\section{Preliminary and notation}

Definition 2.1 A class of linear sequence spaces $E$, called a special space of sequences (sss) having the following conditions:

(1) $E$ is a linear space and $e_{n} \in E$, for each $n \in \mathbb{N}$.

(2) If $x \in \omega, y \in E$ and $\left|x_{n}\right| \leq\left|y_{n}\right|$, for all $n \in \mathbb{N}$, then $x \in E$ 'i.e. $E$ is solid',

(3) if $\left(x_{n}\right)_{n=0}^{\infty} \in E$, then $\left(x_{\left[\frac{n}{2}\right]}\right)_{n=0}^{\infty}=\left(x_{0}, x_{0}, x_{1}, x_{1}, x_{2}, x_{2}, \ldots\right) \in E$, where $\left[\frac{n}{2}\right]$ denotes the integral part of $\frac{n}{2}$.

We call such space $E_{\rho}$ a pre modular special space of sequences if there exists a function $\rho: E \rightarrow[o, \infty[$, satisfies the following conditions:

(i) $\rho(x) \geq 0 \forall x \in E_{\rho}$ and $\rho(\theta)=0$, where $\theta$ is the zero element of $E$,

(ii) there exists a constant $l \geq 1$ such that $\rho(\lambda x) \leq l|\lambda| \rho(x)$ for all values of $x \in E$ and for any scalar $\lambda$,

(iii) for some numbers $k \geq 1$, we have the inequality $\rho(x+y) \leq k(\rho(x)+\rho(y))$, for all $x, y \in E$,

(iv) if $\left|x_{n}\right| \leq\left|y_{n}\right|$, for all $n \in \mathbb{N}$ then $\rho\left(\left(x_{n}\right)\right) \leq \rho\left(\left(y_{n}\right)\right)$,

(v) for some numbers $k_{0} \geq 1$ we have the inequality $\rho\left(\left(x_{n}\right)\right) \leq \rho\left(\left(x_{\left[\frac{n}{2}\right]}\right)\right) \leq k_{0} \rho\left(\left(x_{n}\right)\right)$,

(vi) for each $x=(x(i))_{i=0}^{\infty} \in E$ there exists $s \in \mathbb{N}$ such that $\rho(x(i))_{i=s}^{\infty}<\infty$. This means the set of all finite sequences is $\rho$-dense in $E$.

(vii) for any $\lambda>0$ there exists a constant $\zeta>0$ such that

$$
\rho(\lambda, 0,0,0, \ldots) \geq \zeta \lambda \rho(1,0,0,0, \ldots) .
$$

It is clear that from condition (ii) that $\rho$ is continuous at $\theta$. The function $\rho$ defines a metrizable topology in $E$ endowed with this topology is denoted by $E_{\rho}$.

Example 2.2 $\ell_{p}$ is a pre-modular special space of sequences for $0<p<\infty$, with $\rho(x)=$ $\sum_{n=0}^{\infty}\left|x_{n}\right|^{p}$.

Example 2.3 ces $_{p}$ is a pre-modular special space of sequences for $1<p<\infty$, with $\rho(x)=$ $\sum_{n=0}^{\infty}\left(\frac{1}{n+1} \sum_{k=0}^{n}\left|x_{n}\right|\right)^{p}$.

\section{Definition 2.4}

$$
U_{E}^{\text {app }}:=\left\{U_{E}^{\text {app }}(X, Y) ; X, Y \text { are Banach spaces }\right\}
$$

where

$$
U_{E}^{\mathrm{app}}(X, Y):=\left\{T \in L(X, Y):\left(\alpha_{n}(T)\right)_{n=0}^{\infty} \in E\right\} .
$$

\section{Main results}

Theorem 3.1 $U_{E}^{\text {app }}$ is an operator ideal if $E$ is a special space of sequences (sss).

Proof To prove $U_{E}^{\text {app }}$ is an operator ideal:

(i) let $A \in F(X, Y)$ and $\operatorname{rank}(A)=m$ for all $m \in \mathbb{N}$, since $\mathrm{E}$ is a linear space and $e_{n} \in E$ for each $n \in \mathbb{N}$, then $\left(\alpha_{n}(A)\right)_{n=0}^{\infty}=\left(\alpha_{0}(A), \alpha_{1}(A), \ldots, \alpha_{m-1}(A), 0,0,0, \ldots\right)=$ $\sum_{i=0}^{m-1} \alpha_{i}(A) e_{i} \in E$; for that $A \in U_{E}^{\text {app }}(X, Y)$, which implies $F(X, Y) \subset U_{E}^{\text {app }}(X, Y)$. 
(ii) Let $T_{1}, T_{2} \in U_{E}^{\mathrm{app}}(X, Y)$ and $\lambda_{1}, \lambda_{2} \in \mathbb{R}$ then from Definition 2.1 condition (3) we get $\left(\alpha_{\left[\frac{n}{2}\right]}\left(T_{1}\right)\right)_{n=0}^{\infty} \in E$ and $\left(\alpha_{\left[\frac{n}{2}\right]}\left(T_{2}\right)\right)_{n=0}^{\infty} \in E$, since $n \geq 2\left[\frac{n}{2}\right], \alpha_{n}(T)$ is a decreasing sequence and from the definition of approximation numbers we get

$$
\begin{aligned}
\alpha_{n}\left(\lambda_{1} T_{1}+\lambda_{2} T_{2}\right) & \leq \alpha_{2\left[\frac{n}{2}\right]}\left(\lambda_{1} T_{1}+\lambda_{2} T_{2}\right) \leq \alpha_{\left[\frac{n}{2}\right]}\left(\lambda_{1} T_{1}\right)+\alpha_{\left[\frac{n}{2}\right]}\left(\lambda_{2} T_{2}\right) \\
& \leq\left|\lambda_{1}\right| \alpha_{\left[\frac{n}{2}\right]}\left(T_{1}\right)+\left|\lambda_{2}\right| \alpha_{\left[\frac{n}{2}\right]}\left(T_{2}\right) \quad \text { for each } n \in \mathbb{N} .
\end{aligned}
$$

Since $E$ is a linear space and from Definition 2.1 condition (2) we get $\left(\alpha_{n}\left(\lambda_{1} T_{1}+\lambda_{2} T_{2}\right)\right)_{n=0}^{\infty} \in E$, hence $\lambda_{1} T_{1}+\lambda_{2} T_{2} \in U_{E}^{\text {app }}(X, Y)$.

(iii) If $V \in L\left(X_{0}, X\right), T \in U_{E}^{\mathrm{app}}(X, Y)$ and $R \in L\left(Y, Y_{0}\right)$, then we get $\left(\alpha_{n}(T)\right)_{n=0}^{\infty} \in E$ and since $\alpha_{n}(R T V) \leq\|R\| \alpha_{n}(T)\|V\|$, from Definition 2.1 conditions (1) and (2) we get $\left(\alpha_{n}(R T V)\right)_{n=0}^{\infty} \in E$, then $R T V \in U_{E}^{\mathrm{app}}\left(X_{0}, Y_{0}\right)$.

Theorem 3.2 $U_{\ell_{M}}^{\mathrm{app}}$ is an operator ideal, if $M$ is an Orlicz function satisfying $\Delta_{2}$-condition and there exists a constant $l \geq 1$ such that $M(x+y) \leq l(M(x)+M(y))$.

\section{Proof}

(1-i) Let $x, y \in \ell_{M}$, since $M$ is non-decreasing, we get $\sum_{n=0}^{\infty} M\left(\left|x_{n}+y_{n}\right|\right) \leq l\left[\sum_{n=0}^{\infty} M\left(\left|x_{n}\right|\right)+\sum_{n=0}^{\infty} M\left(\left|y_{n}\right|\right)\right]<\infty$, then $x+y \in \ell_{M}$.

(1-ii) $\lambda \in \mathbb{R}, x \in \ell_{M}$ since $M$ satisfies $\Delta_{2}$-condition, we get $\sum_{n=0}^{\infty} M\left(\left|\lambda x_{n}\right|\right) \leq|\lambda| l \sum_{n=0}^{\infty} M\left(\left|x_{n}\right|\right)<\infty$, for that $\lambda x \in \ell_{M}$, then from (1-i) and (1-ii) $\ell_{M}$ is a linear space over the field of numbers. Also $e_{n} \in \ell_{M}$ for each $n \in \mathbb{N}$ since $\sum_{i=0}^{\infty} M\left(\left|e_{n}(i)\right|\right)=M(1)<\infty$.

(2) Let $\left|x_{n}\right| \leq\left|y_{n}\right|$ for each $n \in \mathbb{N},\left(y_{n}\right)_{n=0}^{\infty} \in \ell_{M}$, since $M$ is none decreasing, then we get $\sum_{n=0}^{\infty} M\left(\left|x_{n}\right|\right) \leq \sum_{n=0}^{\infty} M\left(\left|y_{n}\right|\right)<\infty$, then $\left(x_{n}\right)_{n=0}^{\infty} \in \ell_{M}$.

(3) Let $\left(x_{n}\right)_{n=0}^{\infty} \in \ell_{M}, \sum_{n=0}^{\infty} M\left(\left|x_{\left[\frac{n}{2}\right]}\right|\right) \leq 2 \sum_{n=0}^{\infty} M\left(\left|x_{n}\right|\right)<\infty$, then $\left(x_{\left[\frac{n}{2}\right]}\right)_{n=0}^{\infty} \in \ell_{M}$. Hence, from Theorem 3.1, it follows that $U_{\ell_{M}}^{\text {app }}$ is an operator ideal.

Theorem 3.3 $U_{c e s\left(p_{n}\right)}^{\mathrm{app}}$ is an operator ideal, if $\left(p_{n}\right)$ is an increasing sequence of positive real numbers, $\lim _{n \rightarrow \infty} \sup p_{n}<\infty$ and $\lim _{n \rightarrow \infty} \inf p_{n}>1$.

Proof

(1-i) Let $x, y \in \operatorname{ces}\left(p_{n}\right)$ since

$$
\begin{aligned}
& \sum_{n=0}^{\infty}\left(\frac{1}{n+1} \sum_{k=0}^{n}\left|x_{k}+y_{k}\right|\right)^{p_{n}} \\
& \quad \leq 2^{h-1}\left(\sum_{n=0}^{\infty}\left(\frac{1}{n+1} \sum_{k=0}^{n}\left|x_{k}\right|\right)^{p_{n}}+\sum_{n=0}^{\infty}\left(\frac{1}{n+1} \sum_{k=0}^{n}\left|y_{k}\right|\right)^{p_{n}}\right), \\
& h=\sup _{n} p_{n},
\end{aligned}
$$

then $x+y \in \operatorname{ces}\left(p_{n}\right)$.

(1-ii) Let $\lambda \in \mathbb{R}, x \in \operatorname{ces}\left(p_{n}\right)$, then

$$
\sum_{n=0}^{\infty}\left(\frac{1}{n+1} \sum_{k=0}^{n}\left|\lambda x_{k}\right|\right)^{p_{n}} \leq \sup _{n}|\lambda|^{p_{n}} \sum_{n=0}^{\infty}\left(\frac{1}{n+1} \sum_{k=0}^{n}\left|x_{k}\right|\right)^{p_{n}}<\infty
$$

we get $\lambda x \in \operatorname{ces}\left(p_{n}\right)$, from (1-i) and (1-ii) $\operatorname{ces}\left(p_{n}\right)$ is a linear space. 
To show that $e_{m} \in \operatorname{ces}\left(p_{n}\right)$ for each $m \in \mathbb{N}$, since $\lim _{n \rightarrow \infty} \inf p_{n}>1$ we have $\sum_{n=0}^{\infty}\left(\frac{1}{n+1}\right)^{p_{n}}<$ $\infty$. Thus, we get

$$
\rho\left(e_{m}\right)=\sum_{n=m}^{\infty}\left(\frac{1}{n+1} \sum_{k=0}^{n}\left|e_{m}(k)\right|\right)^{p_{n}}=\sum_{n=m}^{\infty}\left(\frac{1}{n+1}\right)^{p_{n}}<\infty
$$

Hence $e_{m} \in \operatorname{ces}\left(p_{n}\right)$.

(2) Let $\left|x_{n}\right| \leq\left|y_{n}\right|$ for each $n \in \mathbb{N}$, then

$$
\sum_{n=0}^{\infty}\left(\frac{1}{n+1} \sum_{k=0}^{n}\left|\lambda x_{k}\right|\right)^{p_{n}} \leq \sup _{n}|\lambda|^{p_{n}} \sum_{n=0}^{\infty}\left(\frac{1}{n+1} \sum_{k=0}^{n}\left|y_{k}\right|\right)^{p_{n}}<\infty,
$$

since $y \in \operatorname{ces}\left(p_{n}\right)$. Thus, $x \in \operatorname{ces}\left(p_{n}\right)$.

(3) Let $\left(x_{n}\right) \in \operatorname{ces}\left(p_{n}\right)$, then we have

$$
\begin{aligned}
\sum_{n=0}^{\infty} & \left(\frac{1}{n+1} \sum_{k=0}^{n}\left|x_{\left[\frac{k}{2}\right]}\right|\right)^{p_{n}} \\
= & \sum_{n=0}^{\infty}\left(\frac{1}{2 n+1} \sum_{k=0}^{2 n}\left|x_{\left[\frac{k}{2}\right]}\right|\right)^{p_{2 n}}+\sum_{n=0}^{\infty}\left(\frac{1}{2 n+2} \sum_{k=0}^{2 n+1}\left|x_{\left[\frac{k}{2}\right]}\right|\right)^{p_{2 n+1}} \\
= & \sum_{n=0}^{\infty}\left(\frac{1}{2 n+1}\left(\left(\sum_{k=0}^{n} 2\left|x_{k}\right|\right)+\left|x_{n}\right|\right)\right)^{p_{n}}+\sum_{n=0}^{\infty}\left(\frac{1}{2 n+2}\left(\sum_{k=0}^{n} 2\left|x_{k}\right|\right)\right)^{p_{n}} \\
\leq & 2^{h-1}\left(\sum_{n=0}^{\infty}\left(\frac{1}{n+1}\left(2 \sum_{k=0}^{n}\left|x_{k}\right|\right)\right)^{p_{n}}+\sum_{n=0}^{\infty}\left(\frac{1}{n+1} \sum_{k=0}^{n}\left|x_{k}\right|\right)^{p_{n}}\right) \\
& +\sum_{n=0}^{\infty}\left(\frac{1}{n+1} \sum_{k=0}^{n}\left|x_{k}\right|\right)^{p_{n}} \\
\leq & 2^{h-1}\left(2^{h}+1\right) \sum_{n=0}^{\infty}\left(\frac{1}{n+1} \sum_{k=0}^{n}\left|x_{k}\right|\right)^{p_{n}}+\sum_{n=0}^{\infty}\left(\frac{1}{n+1} \sum_{k=0}^{n}\left|x_{k}\right|\right)^{p_{n}} \\
\leq & \left(2^{2 h-1}+2^{h-1}+1\right) \sum_{n=0}^{\infty}\left(\frac{1}{n+1} \sum_{k=0}^{n}\left|x_{k}\right|\right)^{p_{n}}<\infty .
\end{aligned}
$$

Hence, $\left(x_{\left[\frac{n}{2}\right]}\right)_{n=0}^{\infty} \in \operatorname{ces}\left(p_{n}\right)$. Hence, from Theorem 3.1 it follows that $U_{c e s\left(p_{n}\right)}^{\mathrm{app}}$ is an operator ideal.

Theorem 3.4 Let $M$ be an Orlicz function. Then the linear space $F(X, Y)$ is dense in $U_{\ell_{M}}^{\mathrm{app}}(X, Y)$.

Proof Define $\rho(x)=\sum_{n=0}^{\infty} M\left(\left|x_{n}\right|\right)$ on $\ell_{M}$. First we prove that every finite mapping $T \in$ $F(X, Y)$ belongs to $U_{\ell_{M}}^{\text {app }}(X, Y)$. Since $e_{m} \in \ell_{M}$ for each $m \in \mathbb{N}$ and $\ell_{M}$ is a linear space then for every finite mapping $T \in F(X, Y)$ the sequence $\left(\alpha_{n}(T)\right)_{n=0}^{\infty}$ contains only finitely many numbers different from zero. To prove that $U_{\ell_{M}}^{\mathrm{app}}(X, Y) \subseteq \overline{F(X, Y)}$, let $T \in U_{\ell_{M}}^{\mathrm{app}}(X, Y)$, we get $\left(\alpha_{n}(T)\right)_{n=0}^{\infty} \in \ell_{M}$, and since $\sum_{n=0}^{\infty} M\left(\alpha_{n}(T)\right)<\infty$, let $\left.\left.\varepsilon \in\right] 0,1\right]$ then there exists a natural number $s>0$ such that $\sum_{n=s}^{\infty} M\left(\alpha_{n}(T)\right)<\frac{\varepsilon}{4}$, since $\rho$ is none decreasing and $\alpha_{n}(T)$ is 
decreasing for each $n \in \mathbb{N}$, we get

$$
s M\left(\alpha_{2 s}(T)\right) \leq \sum_{n=s+1}^{2 s} M\left(\alpha_{n}(T)\right) \leq \sum_{n=s}^{\infty} M\left(\alpha_{n}(T)\right)<\frac{\varepsilon}{4},
$$

then there exists $A \in F_{2 s}(X, Y), \operatorname{rank}(A) \leq 2 s$ with $M(\|T-A\|)<\frac{\varepsilon}{4 s}$, and by using the conditions of $M$ we get

$$
\begin{aligned}
d(T, A) & =\rho\left(\alpha_{n}(T-A)\right)_{n=0}^{\infty}=\sum_{n=0}^{\infty} M\left(\alpha_{n}(T-A)\right) \\
& =\sum_{n=0}^{3 s-1} M\left(\alpha_{n}(T-A)\right)+\sum_{n=3 s}^{\infty} M\left(\alpha_{n}(T-A)\right) \\
& \leq \sum_{n=0}^{3 s-1} M(\|T-A\|)+\sum_{n=3 s}^{\infty} M\left(\alpha_{n}(T-A)\right) \\
& \leq 3 s M(\|T-A\|)+\sum_{n=s}^{\infty} M\left(\alpha_{n+2 s}(T-A)\right) \\
& \leq 3 s M(\|T-A\|)+\sum_{n=s}^{\infty} M\left(\alpha_{n}(T)\right)<\varepsilon .
\end{aligned}
$$

Corollary 3.5 If $0<p<\infty$ and $\mathrm{M}(t)=t^{p}$, we get $U_{\ell^{p}}^{\mathrm{app}}(X, Y)=\overline{F(X, Y)}$. See [3].

Theorem 3.6 The linear space $F(X, Y)$ is dense in $U_{c e s\left(p_{n}\right)}^{\mathrm{app}}(X, Y)$, if $\left(p_{n}\right)$ is an increasing sequence of positive real numbers with $\lim _{n \rightarrow \infty} \sup p_{n}<\infty$ and $\lim _{n \rightarrow \infty} \inf p_{n}>1$.

Proof First we prove that every finite mapping $T \in F(X, Y)$ belongs to $U_{c e s\left(p_{n}\right)}^{\mathrm{app}}(X, Y)$. Since $e_{m} \in \operatorname{ces}\left(p_{n}\right)$ for each $m \in \mathbb{N}$ and $\operatorname{ces}\left(p_{n}\right)$ is a linear space, then for every finite mapping $T \in F(X, Y)$ i.e. the sequence $\left(\alpha_{n}(T)\right)_{n=0}^{\infty}$ contains only finitely many numbers different from zero. Now we prove that $U_{c e s\left(p_{n}\right)}^{\text {app }}(X, Y) \subseteq \overline{F(X, Y)}$. Since $\lim _{n \rightarrow \infty} \inf p_{n}>1$, we have $\sum_{n=0}^{\infty}\left(\frac{1}{n+1}\right)^{p_{n}}<\infty$, let $T \in U_{c e s\left(p_{n}\right)}^{\text {app }}(X, Y)$ we get $\left(\alpha_{n}(T)\right)_{n=0}^{\infty} \in \operatorname{ces}\left(p_{n}\right)$, and since $\rho\left(\left(\alpha_{n}(T)\right)_{n=0}^{\infty}\right)<\infty$, let $\left.\left.\varepsilon \in\right] 0,1\right]$ then there exists a natural number $s>0$ such that $\rho\left(\left(\alpha_{n}(T)\right)_{n=s}^{\infty}\right)<\frac{\varepsilon}{2^{h+3} \delta c}$ for some $c \geq 1$, where $\delta=\max \left\{1, \sum_{n=s}^{\infty}\left(\frac{1}{n+1}\right)^{p_{n}}\right\}$, since $\alpha_{n}(T)$ is decreasing for each $n \in \mathbb{N}$, we get

$$
\begin{aligned}
\sum_{n=s+1}^{2 s}\left(\frac{1}{n+1} \sum_{k=0}^{n} \alpha_{2 s}(T)\right)^{p_{n}} & \leq \sum_{n=s+1}^{2 s}\left(\frac{1}{n+1} \sum_{k=0}^{n} \alpha_{n}(T)\right)^{p_{n}} \\
& \leq \sum_{n=s}^{\infty}\left(\frac{1}{n+1} \sum_{k=0}^{n} \alpha_{k}(T)\right)^{p_{n}}<\frac{\varepsilon}{2^{h+3} \delta c}
\end{aligned}
$$

then there exists $A \in F_{2 s}(X, Y)$,

$$
\begin{aligned}
\operatorname{rank}(A) \leq 2 s \quad \text { with } \sum_{n=2 s+1}^{3 s}\left(\frac{1}{n+1} \sum_{k=0}^{n}\|T-A\|\right)^{p_{n}} & \leq \sum_{n=s+1}^{2 s}\left(\frac{1}{n+1} \sum_{k=0}^{n}\|T-A\|\right)^{p_{n}} \\
& <\frac{\varepsilon}{2^{h+3} \delta c},
\end{aligned}
$$


and

$$
\sup _{n=s}^{\infty}\left(\sum_{k=0}^{s}\|T-A\|\right)^{p_{n}}<\frac{\varepsilon}{2^{2 h+2} \delta},
$$

since $\alpha_{n}(T)=\inf \{\|T-A\|: A \in L(X, Y)$ and $\operatorname{rank}(A) \leq n\}$. Then there exists a natural number $N>0, A_{N}$ with $\operatorname{rank}\left(A_{N}\right) \leq N$ and $\left\|T-A_{N}\right\| \leq 2 \alpha_{N}(T)$. Since $\alpha_{n}(T) \stackrel{n \rightarrow \infty}{\longrightarrow} 0$, then $\left\|T-A_{N}\right\| \stackrel{N \rightarrow \infty}{\longrightarrow} 0$, so we can take

$$
\sum_{n=0}^{s}\left(\frac{1}{n+1} \sum_{k=0}^{n}\|T-A\|\right)^{p_{n}}<\frac{\varepsilon}{2^{h+3} \delta c}
$$

since $\left(p_{n}\right)$ is an increasing sequence and by using (1), (2), (3) and (4), we get

$$
\begin{aligned}
& d(T, A)=\rho\left(\alpha_{n}(T-A)\right)_{n=0}^{\infty} \\
& =\sum_{n=0}^{3 s-1}\left(\frac{1}{n+1} \sum_{k=0}^{n} \alpha_{k}(T-A)\right)^{p_{n}}+\sum_{n=3 s}^{\infty}\left(\frac{1}{n+1} \sum_{k=0}^{n} \alpha_{k}(T-A)\right)^{p_{n}} \\
& \leq \sum_{n=0}^{3 s}\left(\frac{1}{n+1} \sum_{k=0}^{n}\|T-A\|\right)^{p_{n}}+\sum_{n=s}^{\infty}\left(\frac{1}{n+1} \sum_{k=0}^{n+2 s} \alpha_{k}(T-A)\right)^{p_{n+2 s}} \\
& \leq 3 \sum_{n=0}^{s}\left(\frac{1}{n+1} \sum_{k=0}^{n}\|T-A\|\right)^{p_{n}} \\
& +\sum_{n=s}^{\infty}\left(\frac{1}{n+1} \sum_{k=0}^{2 s-1} \alpha_{k}(T-A)+\frac{1}{n+1} \sum_{k=2 s}^{n+2 s} \alpha_{k}(T-A)\right)^{p_{n}} \\
& \leq 3 \sum_{n=0}^{s}\left(\frac{1}{n+1} \sum_{k=0}^{n}\|T-A\|\right)^{p_{n}} \\
& +2^{h-1}\left(\sum_{n=s}^{\infty}\left(\frac{1}{n+1} \sum_{k=0}^{2 s-1} \alpha_{k}(T-A)\right)^{p_{n}}+\sum_{n=s}^{\infty}\left(\frac{1}{n+1} \sum_{k=2 s}^{n+2 s} \alpha_{k}(T-A)\right)^{p_{n}}\right) \\
& \leq 3 \sum_{n=0}^{s}\left(\frac{1}{n+1} \sum_{k=0}^{n}\|T-A\|\right)^{p_{n}} \\
& +2^{h-1}\left(\sum_{n=s}^{\infty}\left(\frac{1}{n+1} \sum_{k=0}^{2 s-1}\|T-A\|\right)^{p_{n}}+\sum_{n=s}^{\infty}\left(\frac{1}{n+1} \sum_{k=0}^{n} \alpha_{k+2 s}(T-A)\right)^{p_{n}}\right) \\
& \leq 3 \sum_{n=0}^{s}\left(\frac{1}{n+1} \sum_{k=0}^{n}\|T-A\|\right)^{p_{n}}+2^{2 h-1}\left(\sup _{n=s}^{\infty}\left(\sum_{k=0}^{s}\|T-A\|\right)^{p_{n}}\right) \sum_{n=s}^{\infty}\left(\frac{1}{n+1}\right)^{p_{n}} \\
& +2^{h-1} \sum_{n=s}^{\infty}\left(\frac{1}{n+1} \sum_{k=0}^{n} \alpha_{k}(T)\right)^{p_{n}}<\varepsilon .
\end{aligned}
$$

Theorem 3.7 Let $X$ be a normed space, $Y$ a Banach space and $E_{\rho}$ be a pre modular special space of sequences (sss), then $U_{E_{\rho}}^{\mathrm{app}}(X, Y)$ is complete. 
Proof Let $\left(T_{m}\right)$ be a Cauchy sequence in $U_{E_{\rho}}^{\text {app }}(X, Y)$, then by using Definition 2.1 condition (vii) and since $U_{E_{\rho}}^{\mathrm{app}}(X, Y) \subseteq L(X, Y)$, we have

$$
\begin{aligned}
\rho\left(\left(\alpha_{n}\left(T_{i}-T_{j}\right)\right)_{n=0}^{\infty}\right) & \geq \rho\left(\alpha_{0}\left(T_{i}-T_{j}\right), 0,0,0, \ldots\right) \\
& =\rho\left(\left\|T_{i}-T_{j}\right\|, 0,0,0, \ldots\right) \geq \zeta\left\|T_{i}-T_{j}\right\| \rho(1,0,0,0, \ldots),
\end{aligned}
$$

then $\left(T_{m}\right)$ is also Cauchy sequence in $L(X, Y)$. Since the space $L(X, Y)$ is a Banach space, then there exists $T \in L(X, Y)$ such that $\left\|T_{m}-T\right\| \stackrel{m \rightarrow \infty}{\longrightarrow} 0$ and since $\left(\alpha_{n}\left(T_{m}\right)\right)_{n=0}^{\infty} \in E$ for all $m \in \mathbb{N}, \rho$ is continuous at $\theta$ and using Definition 2.1(iii), we have

$$
\begin{aligned}
\rho\left(\alpha_{n}(T)\right)_{n=0}^{\infty} & =\rho\left(\alpha_{n}\left(T-T_{m}+T_{m}\right)\right)_{n=0}^{\infty} \leq k \rho\left(\alpha_{\left[\frac{n}{2}\right]}\left(T_{m}-T\right)\right)_{n=0}^{\infty}+k \rho\left(\alpha_{\left[\frac{n}{2}\right]}\left(T_{m}\right)\right)_{n=0}^{\infty} \\
& \leq k \rho\left(\left(\left\|T_{m}-T\right\|\right)_{n=0}^{\infty}\right)+k \rho\left(\alpha_{n}\left(T_{m}\right)\right)_{n=0}^{\infty}<\varepsilon, \quad \text { for some } k \geq 1 .
\end{aligned}
$$

Hence $\left(\alpha_{n}(T)\right)_{n=0}^{\infty} \in E$ as such $T \in U_{E_{\rho}}^{\mathrm{app}}(X, Y)$.

Corollary 3.8 Let $X$ be a normed space, $Y$ a Banach space and $M$ be an Orlicz function such that $M$ satisfies $\Delta_{2}$-condition. Then $M$ is continuous at $\theta=(0,0,0, \ldots)$ and $U_{\ell_{M}}^{\mathrm{app}}(X, Y)$ is complete.

Corollary 3.9 Let $X$ be a normed space, $Y$ a Banach space and $\left(p_{n}\right)$ be an increasing sequence of positive real numbers with $\lim _{n \rightarrow \infty} \sup p_{n}<\infty$ and $\lim _{n \rightarrow \infty} \inf p_{n}>1$, then $U_{c e s}^{\text {app }}(X, Y)$ is complete.

\section{Competing interests}

The authors declare that they have no competing interests.

\section{Authors' contributions}

NFM gave the idea of the article. AAB carried out the proofs and its application. All authors read and approved the final manuscript.

\section{Author details}

'Department of Mathematics, Faculty of Science, Ain Shams University, Cairo, Egypt. ${ }^{2}$ Department of Mathematics, Faculty of Science and Arts, King Abdulaziz University (KAU), P.O. Box 80200, Khulais, 21589, Saudi Arabia.

\section{Acknowledgements}

Dedicated to Professor Hari M Srivastava.

The authors wish to thank the referees for their careful reading of the paper and for their helpful suggestions.

Received: 7 January 2013 Accepted: 5 April 2013 Published: 18 April 2013

\section{References}

1. Kalton, NJ: Spaces of compact operators. Math. Ann. 208, 267-278 (1974)

2. Lima, $\AA$, Oja, E: Ideals of finite rank operators, intersection properties of balls, and the approximation property. Stud. Math. 133, 175-186 (1999) MR1686696 (2000c:46026)

3. Pietsch, A: Operator Ideals. North-Holland, Amsterdam (1980) MR582655 (81j:47001)

4. Krasnoselskii, MA, Rutickii, YB: Convex Functions and Orlicz Spaces. Noordhoff, Groningen (1961)

5. Orlicz, W: Über Raume ( $\left.L^{M}\right)$. Bull. Int. Acad Polon. Sci. A, 93-107 (1936)

6. Nakano, H: Concave modulars. J. Math. Soc. Jpn. 5, 29-49 (1953)

7. Maddox, IJ: Sequence spaces defined by a modulus. Math. Proc. Camb. Philos. Soc. 100(1), 161-166 (1986)

8. Ruckle, WH: FK spaces in which the sequence of coordinate vectors is bounded. Can. J. Math. 25, $973-978$ (1973)

9. Tripathy, BC, Chandra, P On some generalized difference paranormed sequence spaces associated with multiplier sequences defined by modulus function. Anal. Theory Appl. 27(1), 21-27 (2011)

10. Lindenstrauss, J, Tzafriri, L: On Orlicz sequence spaces. Isr. J. Math. 10, 379-390 (1971)

11. Altin, Y, Et, M, Tripathy, BC: The sequence space $\left|\overline{N_{p}}\right|(M, r, q, s)$ on seminormed spaces. Appl. Math. Comput. 154, 423-430 (2004) 
12. Et, M, Altin, Y, Choudhary, B, Tripathy, BC: On some classes of sequences defined by sequences of Orlicz functions. Math. Inequal. Appl., 9(2), 335-342 (2006)

13. Sanhan, W, Suantai, S: On k-nearly uniformly convex property in generalized Cesáro sequence space. Int. J. Math. Math. Sci. 57, 3599-3607 (2003)

14. Rath, D, Tripathy, BC: Matrix maps on sequence spaces associated with sets of integers. Indian J. Pure Appl. Math. 27(2), 197-206 (1996)

15. Tripathy, BC, Sen, M: On generalized statistically convergent sequences. Indian J. Pure Appl. Math. 32(11), 1689-1694 (2001)

16. Tripathy, BC, Hazarika, B: Paranormed I-convergent sequences. Math. Slovaca 59(4), 485-494 (2009)

17. Tripathy, BC, Sen, M: Characterization of some matrix classes involving paranormed sequence spaces. Tamkang J. Math. 37(2), 155-162 (2006)

18. Tripathy, BC: Matrix transformations between some classes of sequences. J. Math. Anal. Appl. 206, 448-450 (1997)

19. Tripathy, BC: On generalized difference paranormed statistically convergent sequences. Indian J. Pure Appl. Math. 35(5), 655-663 (2004)

20. Altay Band Başar, F: Generalization of the sequence space $\ell(p)$ derived by weighted means. J. Math. Anal. Appl. 330(1), 147-185 (2007)

doi:10.1186/1029-242X-2013-186

Cite this article as: Mohamed and Bakery: Mappings of type Orlicz and generalized Cesáro sequence space. Journal of Inequalities and Applications 2013 2013:186.

\section{Submit your manuscript to a SpringerOpen ${ }^{\circ}$ journal and benefit from:}

- Convenient online submission

Rigorous peer review

- Immediate publication on acceptance

- Open access: articles freely available online

- High visibility within the field

- Retaining the copyright to your article 